\title{
Network Slicing for eMBB and mMTC with NOMA and Space Diversity Reception
}

\author{
Eduardo Noboro Tominaga*, Hirley Alves*, Onel L. Alcaraz López*, Richard Demo Souza ${ }^{\dagger}$, João Luiz \\ Rebelatto ${ }^{\ddagger}$, Matti Latva-aho* \\ *6G Flagship, Centre for Wireless Communications (CWC), University of Oulu, Finland \\ ${ }^{\dagger}$ Federal University of Santa Catarina (UFSC), Florianópolis, Brazil \\ ${ }^{\ddagger}$ Federal University of Technology - Paraná (UTFPR), Curitiba, Brazil \\ \{firstname.lastname\}@oulu.fi, richard.demo@ufsc.br, jlrebelatto@utfpr.edu.br
}

\begin{abstract}
In this work, we study the coexistence in the same Radio Access Network (RAN) of two use cases present in the Fifth Generation (5G) of wireless communication systems: enhanced Mobile BroadBand (eMBB) and massive MachineType Communications (mMTC). eMBB services are requested for applications that demand extremely high data rates and moderate requirements on latency and reliability, whereas mMTC enables applications for connecting a massive number of low-power and low-complexity devices. The coexistence of both services is enabled by means of network slicing and Non-Orthogonal Multiple Access (NOMA) with Successive Interference Cancellation (SIC) decoding. Under the orthogonal slicing, the radio resources are exclusively allocated to each service, while in the non-orthogonal slicing the traffics from both services overlap in the same radio resources. We evaluate the uplink performance of both services in a scenario with a multi-antenna Base Station (BS). Our simulation results show that the performance gains obtained through multiple receive antennas are more accentuated for the non-orthogonal slicing than for the orthogonal allocation of resources, such that the non-orthogonal slicing outperforms its orthogonal counterpart in terms of achievable data rates or number of connected devices as the number of receive antennas increases.
\end{abstract}

Index Terms-5G, eMBB, mMTC, Network Slicing, NOMA, SIC, Spatial Diversity.

\section{INTRODUCTION}

The Fifth Generation (5G) of wireless communications systems features three use cases [1]: enhanced Mobile BroadBand (eMBB), Ultra-Reliable and Low Latency Communications (URLLC) and massive Machine-Type Communications (mMTC). The objective of eMBB is to provide connectivity with extremely high peak data rates and relatively low latency, while at the same time guaranteeing moderate and uniform connectivity over the whole coverage area. URLLC is envisioned for real-time monitoring and control applications with extremely demanding latency and reliability requirements. Finally, mMTC aims at providing ubiquitous connectivity for a massive number of low power and low complexity devices [1].

Despite $5 \mathrm{G}$ is still under standardization and initial phases of deployment around the world, the research community has already started working on the definition of the Key Performance Indicators (KPIs) and development of technical solutions for the Sixth Generation (6G) of wireless communication systems. Regarding the mMTC use cases, it is predicted that the number of connected devices will increase substantially, up to hundreds of devices per cubic meter, which poses extremely challenging requirements on spatial spectral efficiency and required frequency bands for connectivity [2]. One of the use cases for mMTC towards $6 \mathrm{G}$ are the connected industries, where the massive connectivity will enable datadriven solutions for unprecedented levels of customization of products, as well as the improvement of the operation and performance efficiency [3].

Previous generations of wireless communication systems relied mostly on Orthogonal Multiple Access (OMA) schemes. In such schemes, resources that are orthogonal in time and/or frequency are exclusively assigned to different users such that ideally no interference exists among them. However, the major drawback of OMA is that the number of connected devices is limited by the number of available orthogonal radio resources. Meanwhile, Non-Orthogonal Multiple Access (NOMA) techniques have been proposed for the uplink in 5G. NOMA constitues a promising solution to enhance the spectral efficiency and to allow the massive connectivity of users required by mMTC applications. Under NOMA, different users can share the same time/frequency resource through different power allocations or different code signatures. In the case of users with different power levels, the overlapping signals are decoded using Successive Interference Cancellation (SIC) [4].

Another important technique used to enhance the performance of $5 \mathrm{G}$ systems is multiple antennas. Since the separation necessary to ensure independence between antennas decreases with the carrier frequency, for higher-frequencies, e.g. mmWave band, a massive number of antennas may be available, which increases the capability for beamforming. On the other hand, for the lower frequency bands, the number of antennas is typically low to moderate, e.g. up to 32 active antennas [5]. Nonetheless, the available bandwidth in the lower frequency bands is scarce, which may require the combination of multi-antenna techniques with other solutions to increase the number of connected users and the spectral efficiency, e.g. NOMA.

Aiming at allowing the three $5 \mathrm{G}$ use cases to coexist in the same Radio Access Network (RAN) when the number of radio resources is limited, Popovski et. al. [6] proposed a communication-theoretic framework for the slicing of radio 
resources based on the utilization of NOMA techniques. However, they did not consider the use of multiple receive antennas on the Base Station (BS). Other works also studied the coexistence between eMBB and mMTC, e.g. [7], [8] and [9], but being restricted to single antenna receivers as well.

The uplink scenario where multiple MTC devices are allowed to communicate with one or multiple receivers using NOMA schemes has also been studied on other works. In [10], the authors studied the uplink mMTC in a large-scale cellular network overlaid with data aggregators using an analytical framework based on stochastic geometry. In [11], the authors studied a multi-cell scenario with single cell BSs for a UltraNarrow Band (UNB) Low Power Wide Area Network (LPWAN). They considered two different SIC mechanisms: SIC performed locally at each BS without information exchange between BSs, and SIC performed across multiple BSs where BSs can send decoded packets to neighboring cells. The performance of a Long Range (LoRa) network with multiple LoRa devices connected in the uplink with a single antenna BS is studied in [12]. Therein, the BS is allowed to perform a SIC decoding with one iteration to avoid packet losses due to collisions.

In the scope of multiple antenna receivers, Liu et. al. [13] studied the performance of a single-cell large scale MultiUser-MIMO (MU-MIMO) uplink system. They investigated the performance of different linear receivers in a scenario where the users were allowed to communicate with the BS simultaneously in the same time-frequency resource. However, they did not consider the coexistence of devices with heterogeneous performance requirements.

Inspired on the recent works that study MTC uplink scenarios with receive diversity, and specially the setup from [13] and the framework from [6], in this work we study the performance of orthogonal and non-orthogonal network slicing in a single-cell scenario where one eMBB device and multiple MTC devices communicate in the uplink with a multi-antenna BS. Based on the assumption that the number of MTC devices may be much larger than the number of receive antennas, the BS utilizes an iterative Maximum Ration Combining with SIC (MRC-SIC) receiver to decode the multiple signals that arrive simultaneously. Differently from [13], we implement a setup with heterogeneous devices communicating in the uplink. Besides, while in [6] the authors considered only a single-antenna BS, we evaluate the performance gains provided by space diversity reception, i.e. the use of multiple receive antennas. The performance is evaluated in terms of achievable data rates and number of connected MTC devices for given reliability requirements of both services. We show, through Monte Carlo simulations, that despite the space diversity reception improves the performance of both slicing schemes, the performance gains are more accentuated for the non-orthogonal slicing, which makes it a more attractive choice than the orthogonal slicing when the BS is equipped with multiple antennas.

The remainder of this paper is organized as follows. In Section II we present the system model and the performance analysis of eMBB and mMTC when they are considered in isolation. In Section III, we formulate the orthogonal and non-orthogonal slicing of radio resources between eMBB and

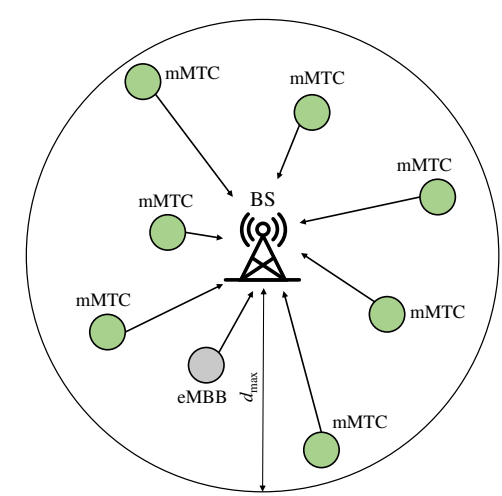

Fig. 1: The uplink of a $5 \mathrm{G}$ network where an eMBB and multiple MTC devices are connected to a common BS.

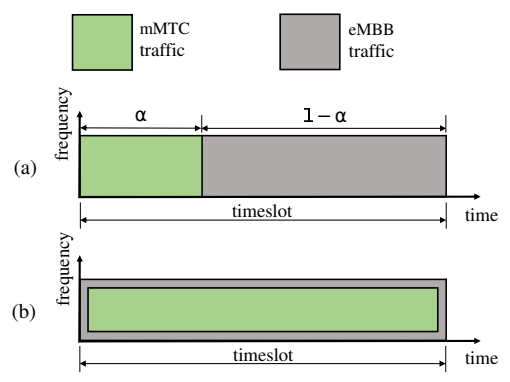

Fig. 2: Orthogonal (a) and non-orthogonal (b) slicing of radio resources between eMBB and mMTC.

mMTC. The Monte Carlo simulation results of both slicing schemes and discussions are presented in Section IV. Finally, we draw our conclusions in Section V.

\section{SYSTEM MODEL}

We analyze the uplink performance of a $5 \mathrm{G}$ network where a single eMBB device and multiple MTC devices transmit independent packets to a common BS, as illustrated in Fig. 1. The eMBB device and each MTC device are single antenna devices, whereas the BS is equipped with $L$ receive antennas, indexed by $l \in\{1, \ldots, L\}$.

We consider a radio resource composed of one timeslot $t$ in a single frequency channel $f$ that can be shared by the eMBB and MTC devices under the orthogonal or non-orthogonal slicing schemes originally proposed in [6] and illustrated in Fig. 2. Under the orthogonal slicing, a fraction $\alpha$ of the radio resource is allocated exclusively to the mMTC traffic, while the remaining part is allocated exclusively to the eMBB traffic. Conversely, the whole radio resource is allocated to eMBB and mMTC under the non-orthogonal slicing, thus there is an overlap of the traffic from both services during the whole timeslot.

As in [6], we assume a standard scheduled transmission phase for the eMBB traffic, where the scheduling of the eMBB device has been solved prior to the considered timeslot. The frequency channel $f$ is assumed to be within the timeand frequency-coherence interval, so that the wireless channel 
coefficients are constant within each timeslot and also fade independently across different timeslots. The wireless channel gains of the eMBB and MTC devices as seen by the receive antenna $l$, which we denote by $g_{i, l}$ with $i \in\{B, M\}$, are independent and identically distributed (i.i.d.) and follow a zero-mean complex Gaussian distribution with variance $\Gamma_{i}$, i.e. $g_{i, l} \sim \mathcal{C N}\left(0, \Gamma_{i}\right)$, where $\Gamma_{i}$ is the average channel gain.

Let us denote by $\mathbf{g}_{i}=\left[g_{i, 1}, \ldots, g_{i, L}\right]^{T}$ the vector of the wireless channel gains from the eMBB or MTC device to the $\mathrm{BS}$ as seen by the $L$ receive antennas. In the case of interference-free transmissions, the received Signal-to-Noise Ratio (SNR) obtained after applying MRC is given by

$$
\gamma_{i}=\left\|\mathbf{g}_{i}\right\|^{2}
$$

In our model, we assume that the average transmit power of all devices is normalized to one. The differences in the actual transmit power of the devices and in the path loss are accounted for through the average channel gains $\Gamma_{i}$. Moreover, we also consider that the noise power at the receiver is normalized to one, such that the average channel gain $\Gamma_{i}$ can be also interpreted as the average received SNR.

No Channel-State Information (CSI) is assumed at the MTC devices, whereas the eMBB device and BS are assumed to have perfect CSI as in [6]. As a consequence, the eMBB device can adapt its transmit power according to the instantaneous channel conditions. Since the MTC devices operate without CSI, they all transmit with the same power.

The outage probabilities of the eMBB and mMTC services are denoted as $\operatorname{Pr}\left(E_{B}\right)$ and $\operatorname{Pr}\left(E_{M}\right)$, respectively, and must satisfy the reliability requirements $\operatorname{Pr}\left(E_{B}\right) \leq \epsilon_{B}$ and $\operatorname{Pr}\left(E_{M}\right) \leq \epsilon_{M}$.

In the following subsections, we present the performance analysis of the eMBB and mMTC services when operating in an orthogonal fashion, by extending the results from [6] to a scenario where the receiver is provided with multiple antennas operating under MRC.

\section{A. Performance Analysis of eMBB}

The eMBB device adapts its transmit power $P_{B}\left(\gamma_{B}\right)$ according to the instantaneous channel gains such that the received SNR always equals a predefined value. Following [6], the objective of the eMBB device is to transmit at the largest rate $r_{B}$ that is compatible with the outage probability requirement $\epsilon_{B}$ under a long-term average power constraint. This can be formulated as the following optimization problem

$$
\begin{aligned}
& \text { maximize } r_{B} \\
& \text { subject to } \operatorname{Pr}\left\{\log _{2}\left[1+P_{B}\left(\gamma_{B}\right) \gamma_{B}\right] \leq r_{B}\right\} \leq \epsilon_{B} \\
& \text { and } \mathbb{E}\left\{P_{B}\left(\gamma_{B}\right)\right\}=1 .
\end{aligned}
$$

The optimal solution to this problem is given by the truncated power inversion scheme: the eMBB device chooses a transmit power that is inversely proportional to the received SNR $\gamma_{B}$ if the latter is above a given threshold $\gamma_{B}^{\mathrm{min}}$, while it refrains from transmitting otherwise [6]. Thus, the activation probability of the eMBB device can be written as [14, Eq. 7.17]

$$
\begin{aligned}
a_{B} & =\operatorname{Pr}\left\{\gamma_{B} \geq \gamma_{B}^{\min }\right\} \\
& =\exp \left(-\frac{\gamma_{B}^{\min }}{\Gamma_{B}}\right) \sum_{l=1}^{L} \frac{\left(\gamma_{B}^{\min } / \Gamma_{B}\right)^{l-1}}{(l-1) !} \\
& =\frac{\Gamma\left(L, \gamma_{B}^{\min } / \Gamma_{B}\right)}{(L-1) !},
\end{aligned}
$$

where $\Gamma(a, z)=\int_{z}^{\infty} t^{a-1} e^{-t} \mathrm{~d} t$ is the upper incomplete gamma function.

In the absence of interference from the mMTC traffic, the only source of outage for an eMBB transmission is the non-transmission event because of extremely poor channel conditions. In this case, the outage probability of the eMBB device can be written as

$$
\operatorname{Pr}\left(E_{B}\right)=\operatorname{Pr}\left\{\gamma_{B}<\gamma_{B}^{\min }\right\}=1-a_{B},
$$

where $a_{B}$ is given by (3).

Imposing the reliability requirement $\operatorname{Pr}\left(E_{B}\right)=\epsilon_{B}$ on (4), we obtain the threshold SNR as

$$
\gamma_{B}^{\min }=\Gamma_{B} \gamma^{-1}\left(L, \epsilon_{B}(L-1) !\right)
$$

where $\gamma(a, z)=\int_{0}^{z} t^{a-1} e^{-t} \mathrm{dt}$ is the lower incomplete gamma function.

Based on the truncated power inversion scheme, the instantaneous power $P_{B}\left(\gamma_{B}\right)$ chosen as a function of the received SNR $\gamma_{B}$ is

$$
P_{B}\left(\gamma_{B}\right)=\left\{\begin{array}{ll}
\frac{\gamma_{B}^{\mathrm{tar}}}{\gamma_{B}} & \text { if } \gamma_{B} \geq \gamma_{B}^{\min } \\
0 & \text { if } \gamma_{B}<\gamma_{B}^{\min }
\end{array},\right.
$$

where $\gamma_{B}^{\mathrm{tar}}$ is the target SNR, which is obtained by imposing the average power constraint as [6]

$$
\begin{aligned}
\mathbb{E}\left\{P_{B}\left(\gamma_{B}\right)\right\} & =\int_{\gamma_{B}^{\min }}^{\infty} \frac{\gamma^{L-1} e^{-\gamma / \Gamma_{B}}}{\Gamma_{B}^{L}(L-1) !} P_{B}(\gamma) d \gamma \\
& =\frac{\gamma_{B}^{\mathrm{tar}}}{\Gamma_{B}(L-1) !} \Gamma\left(L-1, \frac{\gamma_{B}^{\min }}{\Gamma_{B}}\right)=1 .
\end{aligned}
$$

This implies that the target SNR is

$$
\gamma_{B}^{\mathrm{tar}}=\frac{\Gamma_{B}(L-1) !}{\Gamma\left(L-1, \frac{\gamma_{B}^{\min }}{\Gamma_{B}}\right)} .
$$

Finally, the outage rate achieved by the eMBB device is [6]

$$
r_{B}^{\text {out }}=\log _{2}\left(1+\gamma_{B}^{\mathrm{tar}}\right)
$$

\section{B. Performance Analysis of the mMTC}

We assume that $M$ MTC devices are connected to the BS. Following [13] and considering the absence of interference from the eMBB traffic, the $L \times 1$ baseband received vector at the $\mathrm{BS}$ is given by

$$
\mathbf{y}=\sqrt{P_{M}} \mathbf{G}_{M} \mathbf{x}_{M}+\mathbf{n},
$$

where $\mathbf{G}_{M} \in \mathbb{C}^{L \times M}$ is the matrix of channel gains between the MTC devices and the BS, $\sqrt{P_{M}} \mathbf{x}_{M} \in \mathbb{C}^{M \times 1}$ is the vector 
of symbols transmitted by the MTC devices, and $\mathbf{n} \in \mathbb{C}^{L \times 1}$ is the vector of Additive White Gaussian Noise (AWGN) samples with zero mean and unit variance. The $m$-th element of $\mathbf{x}_{M}$, $x_{m}$, is zero if the $m$-th MTC device is not active in the timeslot.

By exploiting the perfect CSI, the BS utilizes a MRC-SIC iterative receiver to decode the signals from the multiple MTC devices that arrive in the same timeslot. Following [13], the received signal vector after the MRC processing is given by

$$
\hat{\mathbf{x}}=\mathbf{G}_{M}^{H} \mathbf{y}=\sqrt{P_{M}} \mathbf{G}_{M}^{H} \mathbf{G}_{M} \mathbf{x}_{M}+\mathbf{G}_{M}^{H} \mathbf{n},
$$

where $\hat{\mathbf{x}} \in \mathbb{C}^{M \times 1}$ and the superscript $H$ indicates the conjugate transpose of the matrix $\mathbf{G}_{M}$.

Let $\hat{x}_{m}$ denote the $m$-th element of the vector $\hat{\mathbf{x}}$, which corresponds to the signal transmitted by the $m$-th MTC device. As in [13], we have

$$
\hat{x}_{m}=\sqrt{P_{M}} \mathbf{g}_{m}^{H} \mathbf{g}_{m} x_{m}+\sqrt{P_{M}} \mathbf{g}_{m}^{H} \sum_{m^{\prime} \neq m}^{M} \mathbf{g}_{m^{\prime}} x_{m^{\prime}}+\mathbf{g}_{m}^{H} \mathbf{n},
$$

where $\mathbf{g}_{m} \in \mathbb{C}^{L \times 1}$ denotes the $m$-th column of the matrix $\mathbf{G}_{M}$. The first term in (12) represents the signal transmitted by the $m$-th MTC device, while the remaining terms represent the interfering signals from other MTC devices and the noise.

Since MTC devices are operate without CSI, they all transmit with the same power and have the same target data rate $r_{M}$. During the MRC-SIC decoding, first the BS detects the strongest device among the active MTC devices, decodes its signal, subtracts its interference from the received signal, proceeds to the second strongest MTC device, and so on. The decoding procedure ends when the decoding of one MTC device fails or after all the active MTC devices are correctly decoded.

The SIC decoding ordering is defined in the descending order of received SNRs of the active MTC devices. Let us denote a SIC decoding ordering $\{1, \ldots, M\}$, such that

$$
\mathbf{g}_{1}^{H} \mathbf{g}_{1} \geq \mathbf{g}_{2}^{H} \mathbf{g}_{2} \geq \ldots \geq \mathbf{g}_{M}^{H} \mathbf{g}_{M} .
$$

The Signal-to-Interference-plus-Noise Ratio (SINR) while decoding the signal from the $m$-th MTC device, and assuming that the MTC devices with indexes $\{1, \ldots, m-1\}$ have already been correctly decoded, reads

$$
\sigma_{m}=\frac{P_{M}\left\|\mathbf{g}_{m}\right\|^{4}}{P_{M} \sum_{m^{\prime}=m+1}^{M}\left|\mathbf{g}_{m}^{H} \mathbf{g}_{m^{\prime}}\right|^{2}+\left\|\mathbf{g}_{m}\right\|^{2}} .
$$

Then, the $m$-th MTC device is correctly decoded if the inequality $\log _{2}\left(1+\sigma_{m}\right) \geq r_{M}$ holds.

\section{SLicing BETWEEN EMBB AND MMTC}

In this section we present the two different network slicing schemes that allow the eMBB and mMTC use cases to coexist in the same RAN.

\section{A. Orthogonal Slicing between $e M B B$ and $m M T C$}

Under the orthogonal slicing, the eMBB device and the MTC devices use the radio resource in a time-sharing manner. Let $\alpha \in[0,1]$ and $1-\alpha$ denote the fraction of time in which the frequency channel is allocated to the eMBB traffic and to the mMTC traffic, respectively. For a given time-sharing factor $\alpha$, the eMBB data rate is [6]

$$
r_{B}=\alpha r_{B}^{\text {out }}
$$

where $r_{B}^{\text {out }}$ is given by (9). Similarly, the mMTC data rate is

$$
r_{M}=(1-\alpha) r_{M}^{\text {out }},
$$

where $r_{M}^{\text {out }}$ is the maximum achievable mMTC data rate in the absence of interference from the eMBB traffic.

To characterize the performance of the orthogonal slicing, for each value of $\alpha$, we set an eMBB data rate according to (21). Then we compute the maximum achievable mMTC data rate $r_{M}$ for which the reliability requirements $\epsilon_{B}$ and $\epsilon_{M}$ are met.

\section{B. Non-Orthogonal Slicing between eMBB and mMTC}

Under the non-orthogonal slicing, the eMBB and mMTC traffics overlap during the whole timeslot. In his case, the received signal vector at the $\mathrm{BS}$ is

$$
\mathbf{y}=\mathbf{G} \mathbf{x}+\mathbf{n},
$$

where

$$
\mathbf{G}=\left[\begin{array}{llll}
\mathbf{g}_{m, 1} & \mathbf{g}_{m, 2} & \cdots & \mathbf{g}_{m, M} \\
\mathbf{g}_{B}
\end{array}\right]
$$

is a matrix containing the channel gains between all the devices and the $\mathrm{BS}, \mathbf{G} \in \mathbb{C}^{L \times(M+1)}$,

$$
\mathbf{x}=\left\{\sqrt{P_{M}}\left[x_{m, 1} x_{m, 2} \ldots x_{m, M}\right] \sqrt{P_{B}} x_{B}\right\}^{T}
$$

is the complex vector containing the transmitted symbols from the MTC devices and from the eMBB device, and $\mathbf{n} \in \mathbb{C}^{L \times 1}$ is the vector containing the noise samples. As in the orthogonal case, the received signal vector after the MRC at the $\mathrm{BS}$ is

$$
\hat{\mathbf{x}}=\mathbf{G}^{H} \mathbf{y}=\mathbf{G}^{H} \mathbf{G} \mathbf{x}+\mathbf{G}^{H} \mathbf{n} .
$$

Let us denote $\hat{x}_{m}$ the element of the vector $\hat{\mathbf{x}}$ corresponding to the signal of the $m$-th MTC device, and $\hat{x}_{B}$ the element of the vector $\hat{\mathbf{x}}$ corresponding to the signal of the eMBB device. They read

$$
\begin{array}{r}
\hat{x}_{m}=\sqrt{P_{M}} \mathbf{g}_{m}^{H} \mathbf{g}_{m} x_{m}+\sqrt{P_{M}} \mathbf{g}_{m}^{H} \sum_{m^{\prime} \neq m}^{M} \mathbf{g}_{m^{\prime}} x_{m^{\prime}}+ \\
\sqrt{P_{B}} \mathbf{g}_{m}^{H} \mathbf{g}_{B} x_{B}+\mathbf{g}_{m}^{H} \mathbf{n}, \\
\hat{x}_{B}=\sqrt{P_{B}} \mathbf{g}_{B}^{H} \mathbf{g}_{B} x_{B}+\sqrt{P_{M}} \mathbf{g}_{B}^{H} \sum_{m=1}^{M} \mathbf{g}_{m} x_{m}+\mathbf{g}_{B}^{H} \mathbf{n} .
\end{array}
$$

Assuming the SIC decoding ordering $\{1, \ldots, M\}$ as in the orthogonal case, the SINR of the $m$-th mMTC in the presence of the eMBB interference reads

$$
\sigma_{m}=\frac{P_{M}\left\|\mathbf{g}_{m}\right\|^{4}}{P_{M} \sum_{m^{\prime}=m+1}^{M}\left|\mathbf{g}_{m}^{H} \mathbf{g}_{m^{\prime}}\right|^{2}+P_{B}\left|\mathbf{g}_{m}^{H} \mathbf{g}_{B}\right|^{2}+\left\|\mathbf{g}_{m}\right\|^{2}} .
$$


As in the orthogonal case, the mMTC is correctly decoded if the inequality $\log _{2}\left(1+\sigma_{m}\right) \geq r_{M}$ holds.

After the correct decoding of the $m$-th MTC device, the BS attempts to decode the eMBB device if it has not been decoded yet. Then the SINR of the eMBB device reads

$$
\sigma_{B}=\frac{P_{B}\left\|\mathbf{g}_{B}\right\|^{4}}{P_{M} \sum_{m^{\prime}=m+1}^{M}\left|\mathbf{g}_{B}^{H} \mathbf{g}_{m^{\prime}}\right|^{2}+\left\|\mathbf{g}_{B}\right\|^{2}} .
$$

For a given data rate $r_{B}$, the eMBB device is correctly decoded if the inequality $\log _{2}\left(1+\sigma_{B}\right) \geq r_{B}$ holds.

Under the orthogonal slicing, the eMBB device adopts a fixed target SNR $\gamma_{B}^{\text {tar }}$ that satisfies the power constraint $\mathbb{E}\left\{P_{B}\left(\gamma_{B}\right)\right\}=1$. On the contrary, aiming to minimize the interference that the eMBB traffic causes to the mMTC traffic, under the non-orthogonal slicing we allow the eMBB device to adopt lower values for the target SNR, which yields the inequality [6]

$$
\gamma_{B}^{\mathrm{tar}} \leq \frac{\Gamma_{B}(L-1) !}{\Gamma\left(L-1, \frac{\gamma_{B}^{\min }}{\Gamma_{B}}\right)} .
$$

Consequently, we have $\mathbb{E}\left\{P_{B}\right\} \leq 1$.

Differently from the orthogonal slicing, the error probability for eMBB has two components in the non-orthogonal case: the probability of the eMBB device does not transmit due to insufficient SNR; and the probability of the eMBB device transmits because it has sufficient SNR, but a decoding error occurs due to the interference from the mMTC traffic. In order to satisfy the same reliability requirement from the orthogonal case, we conservatively assume that the interference from eMBB is always present under the non-orthogonal slicing, that is, $a_{B}=1$. In this case, the error probability for eMBB is

$$
\operatorname{Pr}\left(E_{B}\right)=\operatorname{Pr}\left\{\log _{2}\left(1+\sigma_{B}\right)<r_{B}\right\} .
$$

The SIC decoding procedure for the non-orthogonal slicing runs as follows. Initially, all the MTC devices suffer with the interference from eMBB traffic. First the BS attempts to decode the strongest MTC device. If the decoding succeeds, the signal from the decoded device is subtracted from the received signal, BS attempts do decode the second strongest MTC device, and so on. If the decoding of a MTC device fails, the $\mathrm{BS}$ tries to decode the signal from the eMBB device. If its signal is correctly decoded, the interference from eMBB is subtracted from the received signal, then the BS returns to the decoding of the MTC devices, and the procedure continues as described in Section II-B. Otherwise, if the decoding of the eMBB fails, the SIC decoding ends. The other condition that terminates the SIC decoding procedure is when all the MTC devices are correctly decoded, and so the last step is just the decoding of the eMBB signal without the interference from the mMTC traffic. Note that the step when the eMBB device is decoded is random.

The performance characterization of the non-orthogonal slicing is a two dimensional numerical search: first we set an eMBB data rate $r_{B} \in\left[0, r_{B}^{\text {out }}\right]$ and then compute the maximum number achievable mMTC data rate $r_{M}$ that is adopted for all the MTC devices connected to the BS while still satisfying the reliability requirements $\epsilon_{B}$ and $\epsilon_{M}$; during this computation, we seek for the minimum value of $\gamma_{B}^{\text {tar }}$ that can be adopted by the eMBB device.

\section{NumERICAL RESUltS}

In this section we present Monte Carlo simulation results to illustrate the performance of both orthogonal and nonorthogonal slicing of radio resources between eMBB and mMTC. As the general parameters, we set the reliability requirements $\epsilon_{B}=10^{-3}$ and $\epsilon_{M}=10^{-1}$ for eMBB and mMTC, respectively, and also the average channel gains $\Gamma_{B}=20 \mathrm{~dB}$ and $\Gamma_{M}=5 \mathrm{~dB}$ for eMBB and mMTC, respectively.

In Fig. 3 we plot the pairs of achievable data rates $\left(r_{M}, r_{B}\right)$ for a given number $M=10$ of MTC devices connected to the $\mathrm{BS}$, and for increasing values of receive antennas $L \in\{1,2,4,8,16\}$. Conversely, in Fig. 4 we plot the pairs $\left(M_{\max }, r_{M}\right)$ of maximum number of connected MTC devices versus the eMBB data rate for a given mMTC data rate $r_{M}=0.25$ bits/s/Hz, and also for $L \in\{1,2,4,8,16\}$. The dashed curves correspond to the orthogonal slicing, while the continuous curves correspond to the non-orthogonal slicing.

As observed in both figures, increasing $L$ always increase the performance of the system for both slicing schemes. Meanwhile, we also observe that as we increase the number of receive antennas, the non-orthogonal slicing outperforms more easily the orthogonal slicing both in terms of the maximum achievable mMTC data rate $r_{M}$ and of the maximum number of connected MTC devices $M_{\max }$. For $L=1$ and $L=2$, the orthogonal slicing outperforms the non-orthogonal slicing for the whole range of $r_{B}$. However, as we increase the number of receive antennas to $L \geq 4$, the non-orthogonal slicing becomes increasingly more advantageous over the orthogonal slicing, since it allows us to achieve pairs $\left(r_{M}, r_{B}\right)$ and $\left(M_{\max }, r_{B}\right)$ that are not possible to achieve with the orthogonal slicing.

The curves for the orthogonal slicing are straight lines because $r_{M}$ and $r_{B}$ in Fig. 3 and $M_{\max }$ and $r_{B}$ in Fig. 4 are linearly scaled according to the fraction of the timeslot $\alpha$ that is allocated for each service. On the other hand, the curves for the non-orthogonal cases present a non-linear shape because it is defined according to the level of interference that eMBB causes to mMTC. Starting from $r_{B}=0$, there is no interference from the eMBB traffic, so the mMTC performance for both slicing schemes are the same. Then, when $r_{B}>0$, there is an abrupt reduction in the performance of mMTC because of the presence of interference from eMBB traffic. For the lowest values of $r_{B}$, the interference that the eMBB traffic causes to the mMTC traffic is minimal, so almost all the MTC devices are correctly decoded before the decoding of the eMBB device. As we increase $r_{B}$ to the intermediate values, we also increase the interference from eMBB because the eMBB device has to adopt higher values for the target SNR to meet the target data rate. In this regime, the eMBB device starts to be decoded before some of the MTC devices have been decoded. As a consequence, after the correct decoding of eMBB, some of the MTC devices do not suffer with the interference anymore, and the decrease in the performance of mMTC is very smooth. Finally, for the higher values of $r_{B}$, the eMBB device has to adopt higher values for its target 


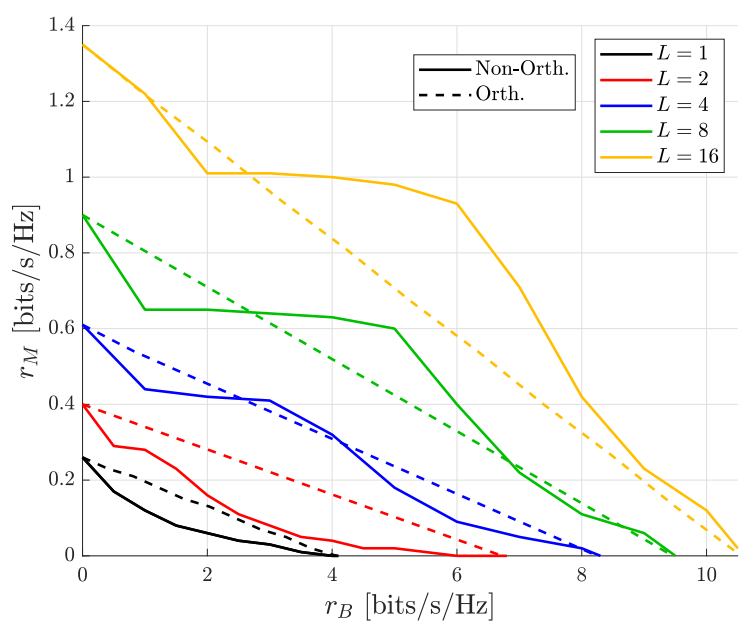

Fig. 3: eMBB data rate $r_{B}$ versus mMTC data rate $r_{M}$ for the orthogonal and non-orthogonal slicing, considering different numbers of receive antennas and for $\Gamma_{B}=20 \mathrm{~dB}, \Gamma_{m}=5 \mathrm{~dB}$, $\epsilon_{B}=10^{-3}, \epsilon_{m}=10^{-1}$ and $M=10$.

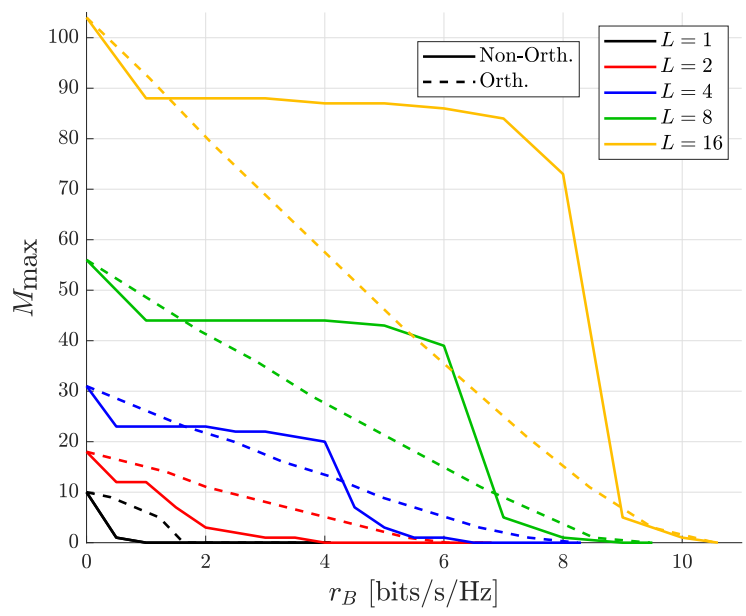

Fig. 4: eMBB data rate $r_{B}$ versus the maximum number of connected MTC devices $M_{\max }$ for the orthogonal and nonorthogonal slicing, considering different numbers of receive antennas and for $\Gamma_{B}=20 \mathrm{~dB}, \Gamma_{m}=5 \mathrm{~dB}, \epsilon_{B}=10^{-3}$, $\epsilon_{m}=10^{-1}$ and $r_{M}=0.25 \mathrm{bits} / \mathrm{s} / \mathrm{Hz}$.

SNR, which causes more interference to the mMTC traffic and makes its performance decrease abruptly. In this regime, the performance of eMBB is also severely limited by the interference from the mMTC traffic.

\section{COnClusions}

We studied the coexistence of eMBB and mMTC in the uplink of the same RAN, enabled by orthogonal and nonorthogonal network slicing schemes. In both schemes, the massive connectivity required by mMTC applications is achieved through the use of NOMA with SIC decoding. The use of multiple receive antennas mitigates the imperfections of the wireless channel and guarantees the spectral efficiency of both services. We set the reliability requirements and then evaluated the pairs of maximum achievable data rates through Monte Carlo simulations. Our simulation results showed that, the more we increase the number of receive antennas, the more advantageous the non-orthogonal slicing becomes over the orthogonal slicing in term of both the achievable mMTC data rates for a given number of connected devices, and the number of connected MTC devices for a given mMTC data rate. Finally, although the spatial receive diversity increases substantially the performance of the system, it also increases the receiver complexity. Moreover, NOMA of a massive number of devices is also a complex task and yields higher processing delay times. Such aspects must be considered in practical implementations.

\section{ACKNOWLEDGMENT}

This research was financially supported by 6Genesis Flagship project (grant no. 318927), FIREMAN project (grant no. 326201) and Academy Professor project from Academy of Finland (grant no. 307492).

\section{REFERENCES}

[1] Ericsson, "5G Systems," Tech. Rep., 2017. [Online]. Available: https://www.ericsson.com/en/reports-and-papers/white-papers/5gsystems--enabling-the-transformation-of-industry-and-society

[2] M. Latva-Aho and K. Leppänen, "Key Drivers and Research Challenges for $6 \mathrm{G}$ Ubiquitous Wireless Intelligence," in $6 G$ Wireless Summit, Levi, Finland, Mar 2019.

[3] N. H. Mahmood, O. López, O.-S. Park, I. Moerman, K. Mikhaylov, E. Mercier, A. Munari, F. Clazzer, S. Böcker, and H. Bartz, "White Paper on Critical and Massive Machine Type Communications Towards 6G," University of Oulu, White Paper, 2020.

[4] S. Lien, S. Shieh, Y. Huang, B. Su, Y. Hsu, and H. Wei, "5G New Radio: Waveform, Frame Structure, Multiple Access, and Initial Access," IEEE Commun. Mag., vol. 55, no. 6, pp. 64-71, 2017.

[5] A. A. Zaid, R. Baldemair, M. Andersson, S. Faxér, V. Molés-Cases, and Z. Wang, "Designing for the Future: the 5G NR Physical Layer," Ericsson Tech. Rev., Jun 2017. [Online]. Available: https://www.ericsson.com/en/reports-and-papers/ericsson-Technol.review/articles/designing-for-the-future-the-5g-nr-physical-layer

[6] P. Popovski, K. F. Trillingsgaard, O. Simeone, and G. Durisi, "5G Wireless Network Slicing for eMBB, URLLC, and mMTC: A Communication-Theoretic View," IEEE Access, vol. 6, pp. 5576555779,2018

[7] O. Vikhrova, C. Suraci, A. Tropeano, S. Pizzi, K. Samouylov, and G. Araniti, "Enhanced Radio Access Procedure in Sliced 5G Networks," in 2019 11th Int. Congr. Ultra Modern Telecommun. Control Syst. Workshops (ICUMT), 2019, pp. 1-6.

[8] N. H. Mahmood, M. Lauridsen, G. Berardinelli, D. Catania, and P. Mogensen, "Radio Resource Management Techniques for eMBB and mMTC Services in 5G Dense Small Cell Scenarios," in 2016 IEEE 84th Veh. Technol. Conf. (VTC-Fall), 2016, pp. 1-5.

[9] M. Kamel, W. Hamouda, and A. Youssef, "Uplink Performance of NOMA-Based Combined HTC and MTC in Ultradense Networks," IEEE Internet Things J., vol. 7, no. 8, pp. 7319-7333, 2020.

[10] O. L. Alcaraz López, H. Alves, P. H. Juliano Nardelli, and M. Latva-aho, "Aggregation and Resource Scheduling in Machine-Type Communication Networks: A Stochastic Geometry Approach," IEEE Trans. Wireless Commun., vol. 17, no. 7, pp. 4750-4765, 2018.

[11] Y. Mo, C. Goursaud, and J. Gorce, "Uplink Multiple Base Stations Diversity for UNB based IoT networks," in 2018 IEEE Conf. Antenna Meas. Appl. (CAMA), 2018, pp. 1-4.

[12] J. M. d. S. Sant'Ana, A. Hoeller, R. D. Souza, H. Alves, and S. MontejoSánchez, "LORA Performance Analysis with Superposed Signal Decoding," IEEE Wireless Commun. Lett., pp. 1-1, 2020.

[13] M. Liu, J. Zhang, C. Xu, P. Zhang, and Y. Wu, "Non-Asymptotic Outage Probability of Large-Scale MU-MIMO Systems with Linear Receivers," in 2016 IEEE 84th Veh. Technol. Conf. (VTC-Fall), 2016, pp. 1-5.

[14] A. Goldsmith, Wireless Communications. Cambridge Univ. Press, 2005. 